\section{O modelo lógico como ferramenta de planejamento, implantação e avaliação do programa de Promoção da saúde na estratégia de saúde da família do Distrito Federal}

\author{
The logic model as a planning, implementation \\ and evaluation tool of a health promotion \\ program on the health family strategy of \\ Distrito Federal
}

Caroline Romeiro

Júlia Aparecida Devidé Nogueira ${ }^{2 *}$

Sarah Guerra Tinoco ${ }^{3}$

Kênia Mara Baiocchi de Carvalho ${ }^{4}$

\section{Resumo}

Esse trabalho visa registrar o desenvolvimento de um programa de Promoção da saúde (PS) na Estratégia de Saúde da Família (ESF) através da elaboração do modelo lógico (ML), com ênfase no planejamento e implantação em seu primeiro ano de funcionamento. A metodologia de elaboração do ML permitiu identificar e registrar os objetivos do programa e as atividades, recursos e estratégias necessários para alcançá-los. A Secretaria de Estado de Saúde do Distrito Federal (DF) idealizou o programa e estabeleceu parcerias internas e externas com universidades e centros de pesquisa para sua implantação na região de São Sebastião-DF. Os profissionais de saúde da ESF local foram sensibilizados e 100\% deles foram capacitados, permitindo que a rede de serviço oferecesse aos usuários oportunidades de adotar estilos de vida saudáveis com ênfase na alimentação e atividade física. Adultos com excesso de peso cadastrados na ESF local foram convidados a participar das atividades, e 279 usuários tiveram seus dados coletados e foram acompanhados nas ações desenvolvidas (13 oficinas multidisciplinares e atividade física regular). A maior adesão registrada foi $61,1 \%$ na oficina de nutrição e a menor foi $11,6 \%$ no grupo de apoio motivacional. $\mathrm{O}$ uso do ML permitiu registrar o planejamento; refinar objetivos; acompanhar a implantação; identificar ações sobrepostas, barreiras e facilitadores; adequar a proposta à realidade encontrada; e definir metas e variáveis avaliativas. A construção do ML reforçou o papel da intersetorialidade no desenvolvimento de programas de PS, otimizou seu funcionamento e irá facilitar sua replicação e expansão em realidades semelhantes.

\section{Palavras-chave}

Sistema Único de Saúde; Planejamento; Avaliação; Comunicação em saúde.

\begin{abstract}
This study aims to report the development of a health promotion project in the Family Health Strategy (ESF) by developing the logical model (ML), with emphasis in planning and implementation during it's first working year. The methodology of developing the ML identified project goals and activities, and resources and strategies needed to achieve them. The State Department of Health of the Federal District (DF) conceived the project and established internal and external partnerships with universities and research centers for its development in the region of São Sebastião-DF. Health professionals of local ESF were sensitized and $100 \%$ of them were trained, enabling service to offer users opportunities to adopt healthy lifestyles with emphasis on diet and physical activity. Overweight adults enrolled in the local ESF were invited to participate and 279 users had their data collected and were followed thought the actions taken during the year $13 \mathrm{mul}$ tidisciplinary workshops and regular physical activity options). The greater adherence recorded was $61.1 \%$ in nutrition workshop and the lowest adherence was $11.6 \%$ in motivational support group. The use of $M L$ has enabled to register the planning, refine goals, monitor implementation, identify overlapping actions, barriers and facilitators, tailor the proposal to the actual situation, and define evaluative goals and variables. The $M L$ development strengthened the role of intersectoral cooperation in the development of health promoting projects; improved its operation and will facilitate its replication and expansion in similar settings.
\end{abstract}

Keywords

Unified Health System; Planning; Evaluation; Communication in health.
Rev Bras Ativ Fis Saúde p. 132-142 DOI: http://dx.doi.org/10.12820/23171634.2013v18n1p132

1 Pós-Graduação em Nutrição Humana, Departamento de Nutrição, Universidade de Brasilia. Brasília - DF. Brasil.

2 Faculdade de Educação Física, Universidade de Brasilia, Brasilia - DF. Brasil.

3 Secretaria de Estado de Saúde do Distrito Federal, Núcleo de Vigilância Epidemiológica de Doenças Não Transmissíveis. Brasília - DF. Brasil.

4 Departamento de Nutrição, Universidade de Brasília. Brasília - DF. Brasil. 


\section{INTRODUÇÃO}

O Brasil enfrenta as transições demográfica, nutricional e epidemiológica; com aumento expressivo da morbi-mortalidade por doenças crônicas não transmissíveis $(\mathrm{DCNT})^{1,2}$. No Distrito Federal (DF), 49,1\% da população apresenta excesso de peso; $24 \%$ consome o recomendado de frutas e hortaliças; $32,8 \%$ pratica atividade física suficiente no tempo livre; e 20,2\% auto-reportam ter hipertensão e 4,7\%, diabetes $^{3}$. Este é um contexto sócio-sanitário que estimula a reorientação das políticas, modelos de atenção e práticas no Sistema Único de Saúde (SUS) para a perspectiva da promoção da Saúde (PS $)^{4-7}$. A PS é uma proposta complexa e abrangente que requer o envolvimento da população, de diversos setores sociais e do Estado para melhorar as condições de saúde e qualidade de vida através de estratégias e ações consonantes com seus princípios: concepção holística, multi-estratégia, intersetorialidade, empoderamento, participação social, equidade e sustentabilidade ${ }^{4}$.

Um dos primeiros passos para desenvolver a PS no SUS foi a implantação da Estratégia Saúde da Família (ESF) em 1994, visando garantir o cuidado integral e reorganizar a atenção básica através de equipes multiprofissionais como elemento de logística5 ${ }^{5}$ Em 2006, a Política Nacional de PS (PNPS) institucionalizou a PS através de diretrizes integradas para garantir, entre outros, hábitos alimentares saudáveis e atividades físicas/práticas corporais sustentáveis como eixos prioritários no cuidado à saúde ${ }^{1,4}$. Outro passo importante na reorientação dos serviços foi, em 2008, a criação do Núcleo de Apoio à Saúde da Família (NASF) favorecendo a formação de equipes multidisciplinares e redes de suporte social ${ }^{6}$. E em 2011 foi lançado o Programa Academia da Saúde visando implantar polos com infraestrutura, equipamentos e profissionais para orientar modos de vida saudáveis, com ênfase nas práticas corporais/atividades físicas, de forma coordenada com os NASF${ }^{7}$.

A recente e rápida institucionalização da PS no SUS criou a demanda pela execução de programas e produção de conhecimentos (avaliações e estudos) nesta nova perspectiva ${ }^{8}$. Entretanto, desenvolver programas em consonância com os princípios de PS é desafiador devido à complexidade no planejamento, descrição, desenvolvimento, avaliação e comunicação das ações ${ }^{1,4,8,9}$. Ademais, apesar da avaliação ser um conceito chave em PS, sua realização em nível comunitário continua sendo um desafio devido à abrangência das intervenções e ao menor controle das variáveis intervenientes nos desfechos ${ }^{10}$, resultando em poucos artigos científicos que avaliaram iniciativas de PS no SUS ${ }^{10-14}$.

Como a sistematização, monitoramento e avaliação são essenciais para favorecer a efetividade, sustentabilidade e compartilhamento de informações dos programas $^{9,10}$, um primeiro passo deve ser a observação e registro do processo em um modelo de avaliação formativa que descreva as intervenções na perspectiva da realidade local ${ }^{10}$, permitindo a compreensão da iniciativa e seu adequado monitoramento ${ }^{10,15}$. Um instrumento que pode facilitar este processo é o Modelo Lógico $(\mathrm{ML})^{9}$. O ML fornece uma linguagem comum entre os responsáveis pelas ações e ajuda a identificar variáveis relevantes, sendo uma ferramenta importante para documentar a descrição e análise dos fatores contextuais do problema a ser enfrentado; da estrutura e dos componentes centrais do programa; das conexões entre estes componentes; das atividades e recursos previstos; e dos resultados esperados ${ }^{9,15}$.

Embora o conhecimento sobre intervenções de PS mereça ser amplamente compartilhado, iniciativas locais têm carecido de adequada divulgação ${ }^{15}$. Assim, o presente estudo decorre do interesse científico somado à necessidade sócio-política de registro e avaliação de programas comunitários que incluam uma descrição 
e registro sistematizado do processo de implantação em âmbito local. O objetivo deste artigo é descrever o programa multidisciplinar de PS, com ênfase na alimentação saudável e atividade física, desenvolvido no DF, através da elaboração do ML com registro do planejamento, implantação e desenvolvimento das ações no decorrer do primeiro ano de trabalho.

\section{MÉTODOS}

Este estudo foi aprovado pelo comitê de ética em pesquisa da Secretaria de Estado de Saúde (SES)/DF, registro n²07/08. Apesar do artigo ter caráter descritivo, é importante informar que o estudo como um todo foi realizado dentro dos padrões éticos exigidos pela resolução 196/96 do Ministério da Saúde.

Para realizar o registro sistemático do processo de implantação, utilizou-se como referência a abordagem de avaliação orientada pela teoria do programa ${ }^{10}$, na qual os avaliadores partem da "teoria" do programa para identificar o que se espera e como se espera alcançar ${ }^{10}$, através da articulação e descrição das ideias, hipóteses e expectativas que constituem a estrutura do programa e seu funcionamento ${ }^{10,15}$. Utilizou-se como ferramenta de orientação o "Manual para Avaliação de Programas de Atividade Física" ", para realizar o registro detalhado do programa de PS e prevenção de DCNT da SES/DF (chamado popularmente de Jogo de Cintura). Este manual prevê seis etapas para avaliação dos programas: 1) obter participação das partes interessadas; 2) descrever o plano do programa; 3) focalizar a avaliação; 4) coletar provas fidedignas; 5) fundamentar as conclusões; e 6) aplicar e compartilhar as lições aprendidas ${ }^{9}$. Como esta é uma experiência pioneira de implantação do programa de PS no DF, o desenvolvimento do presente artigo deu destaque às etapas iniciais - identificação das partes interessadas, elaboração e descrição do programa através do ML, bem como ao compartilhamento das informações sistematizadas e lições aprendidas.

$\mathrm{O}$ desenvolvimento do $\mathrm{ML}$ ocorreu nas reuniões quinzenais do grupo gestor (GG) coordenadas pelo Núcleo de Vigilância Epidemiológica de Doenças Não Transmissíveis (NVEDNT) da SES-DF. As reuniões visavam discutir, planejar e acompanhar as ações do programa, e tinham duração aproximada de duas horas. A versão final do ML foi obtida depois do término do primeiro ano do programa, após aprovação do GG.

\section{RESULTADOS}

O ML apresentado (Figura 1) é entendido como uma representação gráfica de estrutura dinâmica, adaptada durante a execução do programa para garantir adequação com a realidade encontrada. Este ML fornece uma ideia geral do programa explicitando seus objetivos (iniciais, intermediários e finais); recursos utilizados e atividades desenvolvidas a partir da perspectiva do GG; e será utilizado para nortear os futuros processos de avaliação e expansão do programa.

\section{A gênese do programa}

No DF a integração entre as iniciativas de PS e as práticas da ESF vem sendo realizadas de forma coordenada desde 2007, quando foi publicado o Plano Distrital de $\mathrm{PS}^{16}$. Este processo recebeu reforços quando o Ministério da Saúde lançou editais destinando recursos financeiros para estados e municípios que desenvolvessem programas nas áreas prioritárias da $\mathrm{PNPS}^{17}$. Nesse contexto, no ano de 


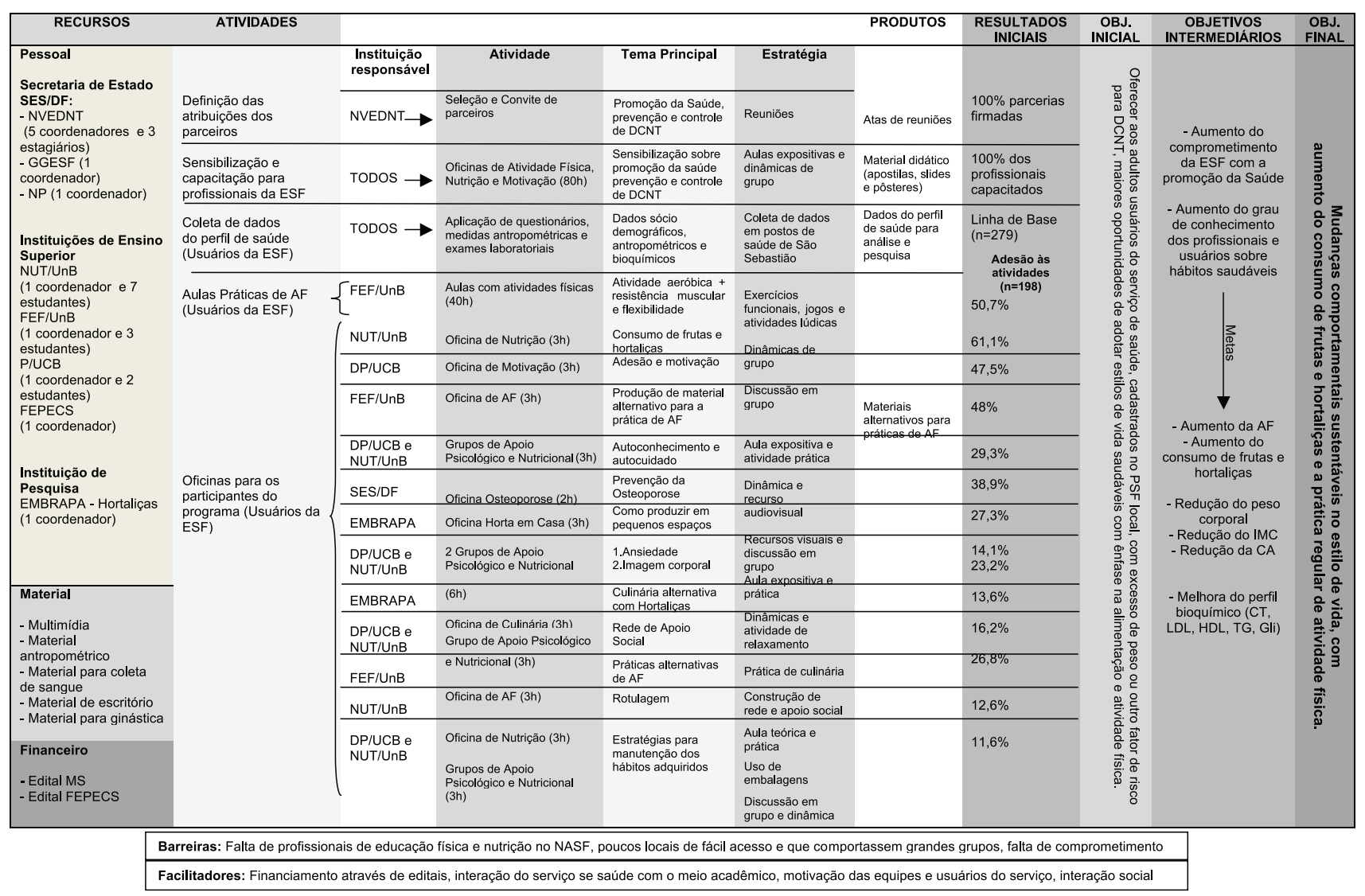

Obj, objetivo; SES/DF, Secretaria de Estado de Saúde do Distrito Federal; NVEDNT, Núcleo de Vigilância Epidemiológica em Doenças e Agravos Não Transmissíveis; GGESF, Gerência de Gestão e Estratégia de Saúde da Família; NP, Núcleo de Patologia; NUT/UnB, Departamento de Nutrição da Universidade de Brasília; FEF/UnB, Faculdade de Educação Física da Universidade de Brasília; DP/UCB, Departamento de Psicologia da Universidade Católica de Brasília; FEPECS, Fundação de Ensino e Pesquisa em Ciências da Saúde; EMBRAPA, Empresa Brasileira de Produtos Agropecuários; MS, Ministério da Saúde; ESF, Estratégia Saúde da Família; NASF, Núcleo de Apoio à Saúde da Família; ACS, Agente Comunitário de Saúde; AF, Atividade Física; DCNT, Doenças Crônicas Não Transmissíveis; CT, Colesterol Total; LDL, Lipoproteína de baixa densidade; TG, Triglicerídeos; Gli, Glicemia; IMC, Índice de Massa Corporal; CA, Circunferência Abdominal.

Figura 1 - Modelo Lógico do Programa Jogo de Cintura.

2009 o programa de incentivo à PS e prevenção de DCNT, o Jogo de Cintura, foi idealizado e passou a ser coordenado pelo NVEDNT/SES-DF.

Como expresso no manual do ML, o primeiro passo para o desenvolvimento de um programa de PS é a mobilização das partes interessadas e tomadores de decisão. No Jogo de Cintura, o NVEDNT/SES-DF articulou as parcerias externas: Universidade de Brasília, Universidade Católica de Brasília, Empresa Brasileira de Produtos Agropecuários (EMBRAPA) e Fundação de Ensino e Pesquisa em Ciências da Saúde (FEPECS); e internas à SES-DF: Conselho de Saúde, Gerência de Gestão e Estratégia da Saúde da Família para implantar um NASF em São Sebastião, e Núcleo de Patologia para realizar os exames laboratoriais. Todas as instituições convidadas se tornaram parceiras e indicaram um coordenador para compor o GG, que passou a se reunir quinzenalmente para discutir e definir aspectos técnicos, elaborar e detalhar o planejamento, atividades e material necessários em cada intervenção. Outra atividade fundamental do GG nestas reuniões foi o registro destas informações no ML.

\section{Os objetivos do programa}

Um ponto central para desenvolver qualquer programa é definir seus objetivos a curto, médio e longo prazo ${ }^{10,15}$. Quando a NVEDNT/SES-DF procurou os parceiros do programa, já trazia uma expectativa de objetivo geral a ser atingido. 
Entretanto, a diversidade do GG gerou impasses e dificuldades de alinhamento conceitual, e dúvidas quanto aos objetivos e resultados esperados. As diferentes percepções foram expressas e o exercício de elaboração do ML auxiliou a obtenção de um entendimento comum sobre o programa e a definição de seus objetivos.

Assim, o objetivo inicial do Jogo de Cintura foi estruturar uma rede de serviço e apoio capaz de oferecer aos usuários maiores oportunidades de adotar estilos de vida saudáveis. O objetivo intermediário foi aumentar o grau de conhecimento e prática dos profissionais e usuários sobre hábitos saudáveis e foi relacionado ao alcance de metas específicas: aumento da média de atividade física para 150 minutos por semana ${ }^{18}$; aumento do consumo médio de frutas e hortaliças para 5 dias da semana ${ }^{19}$; redução média de $5 \%$ do peso corporal ${ }^{20}$; e melhora do perfil bioquímico (colesterol total, colesterol LDL, colesterol HDL, triglicerídeos e glicemia) para níveis saudáveis ${ }^{21,22}$. O objetivo final é fazer com que estas mudanças nos indicadores de saúde e no estilo de vida sejam contínuas, duradouras e sustentáveis. O planejamento e elaboração do ML permitiu identificar recursos que deveriam ser mobilizados e atividades e estratégias que deveriam ser realizadas para alcançar os objetivos; bem como definir a área e a população que o programa deveria atender.

\section{Área de atuação, população e amostra}

Sendo um programa piloto, seu desenvolvimento deveria ocorrer numa área com características que justificassem a prioridade de implantação. O GG indicou a região administrativa de São Sebastião-DF por esta apresentar ESF consolidada (maior cobertura do DF) ${ }^{23}$; índice de desenvolvimento humano (IDH) menor do que a média do $\mathrm{DF}^{24}$; e ter dados publicados indicando elevada prevalência de fatores de risco para $\mathrm{DCNT}^{25}$. São Sebastião possui seis Unidades Básicas de Saúde (UBS), distribuídas em sub-regiões de atendimento, sendo que todas apresentavam ESF consolidada. As UBS foram divididas em grupo intervenção $(n=3)$ e controle $(n=3)$, respeitando-se a localização geográfica para minimizar o contato entre profissionais e usuários dos dois grupos. Análises futuras irão comparar os resultados do grupo controle versus intervenção, mas fogem do escopo do presente artigo.

Para definir a população-alvo ponderou-se vantagens e desvantagens das abordagens básicas de prevenção, a populacional ou a de risco ${ }^{15}$. Optou-se pela abordagem de risco para DCNT e os critérios de inclusão foram definidos considerando o excesso de peso como fator predisponente. Foram selecionados indivíduos de ambos os sexos; adultos (idade entre 18 e 79 anos); com Índice de Massa Corporal (IMC $\geq 25 \mathrm{Kg} / \mathrm{m}^{2}$ ) e/ou circunferência abdominal (CA $\geq 94 \mathrm{~cm}$ para homens e $\geq 80 \mathrm{~cm}$ para mulheres) elevados; que buscaram atendimento na UBS no último ano. Os critérios de exclusão foram: residir fora da região de São Sebastião-DF; estar gestante; usar medicações antiobesidade; e/ou ser portador de neoplasias ou diabetes mellitus tipo I. O cadastro de usuários mantido pela ESF foi utilizado para identificar estes indivíduos. Após a aplicação dos critérios de inclusão e exclusão, chegou-se a um total de 280 indivíduos que foram convidados a participar. Destes, 279 assinaram o TCLE e foram incluídos na pesquisa, sendo que 198 foram atendidos nas UBS do grupo intervenção (assistência padrão da ESF acrescida das intervenções do Jogo de Cintura) e 89 foram atendidos nas UBS do grupo controle (assistência padrão da ESF).

\section{Recursos e atividades}

Os recursos para a implantação do programa foram divididos em: humano, financeiro e material. Os recursos humanos foram compostos por 10 representantes 
da SES-DF e 17 representantes de instituições parceiras do programa (professores e estudantes de graduação em Educação Física, Nutrição e Psicologia das instituições parceiras). Os recursos materiais - equipamento multimídia, material antropométrico (balanças e fitas métricas), material para coleta de sangue, material de escritório (papelaria e livros técnicos), equipamento para ginástica (colchonetes), serviços para impressão de pôsteres e brochuras informativos, e 3 bolsas de monitoria - foram pagos com os recursos financeiros recebidos do Ministério da Saúde ${ }^{17}$ e do edital da FEPECS (no 57, de 29 de outubro de 2008). As atividades desenvolvidas no campo foram divididas em:

a) Intervenções: desenvolvidas pelo GG com conteúdo e dinâmicas traduzindo recomendações de órgãos oficiais para uma linguagem acessível à população, com inclusão de figuras ilustrativas visando atingir os não alfabetizados. As orientações dietéticas seguiram as recomendações do Ministério da Saúde ${ }^{19}$ e da Organização Mundial da Saúde ${ }^{18,27}$, estando centradas na qualidade das escolhas alimentares, principalmente no aumento do consumo de frutas e hortaliças. As orientações de atividade física seguiram as recomendações da Organização Mundial da Saúde ${ }^{18}$, onde a meta estabelecida foi atingir o mínimo de 30 minutos diários em pelo menos cinco dias semanais, de forma contínua ou intervalada, no tempo livre ou no deslocamento.

A partir do material didático desenvolvido (apostilas, slides e pôsteres), os profissionais das seis ESF - médicos, enfermeiros e agentes comunitários de saúde (ACS) - foram sensibilizados e convidados a participar da capacitação multidisciplinar em PS, com emissão de diploma (total de 80 horas nos temas motivação, alimentação saudável, atividade física e PS), visando preparar os profissionais para atuar junto ao programa, inclusive na coleta de dados dos usuários. A capacitação foi conduzida por professores e alunos de graduação em nutrição, educação física e psicologia através de atividades teórico-práticas em grupo e baseada no compartilhamento do saber interdisciplinar, e todos os 52 profissionais aderiram e completaram a capacitação.

Após esta etapa, o programa passou à intervenção junto aos usuários das três ESF pertencentes ao grupo intervenção. Nos oito meses subsequentes foram desenvolvidas oficinas educativas em grupo de caráter teórico-prático, além opções regulares de atividade física. Ao todo foram 13 oficinas multidisciplinares (carga total: 38 horas) abordando os temas de motivação e apoio psicológico, alimentação saudável, culinária, horta em casa, aproveitamento integral dos alimentos, rotulagem de alimentos, atividade física, e produção de materiais alternativos para uso coletivo. Foram oferecidas atividades físicas regulares caminhada todos os dias em dois horários (manhã e tarde) com coordenação do ACS e ginástica (três vezes na semana em dois horários pela tarde) desenvolvidas por 3 alunos de educação física, sob supervisão de um professor formado. Ao final de cada encontro os participantes foram estimulados a descrever dificuldades e avanços em relação às atividades propostas.

b) Coleta de dados: para avaliar o impacto da intervenção na saúde dos participantes, realizou-se a coleta de dados dos usuários antes (março), no meio (junho) e ao final (novembro) do período de intervenções. As coletas de dados foram uma ação conjunta entre profissionais da ESF, parceiros e coordenadores do GG no regime de mutirão. Os instrumentos avaliativos foram de baixa complexidade e custo; dados sócio-demográficos foram obtidos com base em questionário próprio; a atividade física foi avaliada pelo Questionário Interna- 
cional de Atividade Física versão longa ${ }^{26}$, aplicado por estudantes de educação física treinados; e o consumo alimentar foi avaliado pelo questionário de frequência de consumo alimentar ${ }^{3,25}$, aplicado por estudantes de nutrição treinados. Foram ainda realizadas medidas antropométricas e bioquímicas; estatura, peso e CA foram mensuradas de acordo com normas de antropometria e o IMC foi calculado para classificação do estado nutricional ${ }^{27}$; amostras de sangue foram coletadas em jejum para análises de glicemia, triglicerídeos, colesterol total, LDL-colesterol e HDL-colesterol, seguindo protocolo do laboratório da regional de saúde de São Sebastião.

\section{Processo de implantação}

Uma das primeiras análises sobre o processo de implantação do programa foi a adesão dos parceiros à proposta. Todas as instituições convidadas a compor o GG e $100 \%$ dos profissionais das ESF aceitaram participar, o que facilitou a implantação do Jogo de Cintura na rede de serviço. Outro indicador foi a adesão dos usuários às diversas atividades oferecidas ( $99,6 \%$ no primeiro, $70,0 \%$ no segundo e $49,3 \%$ no terceiro mutirão; $61,1 \%$ na oficina de nutrição; $50,7 \%$ nas atividades físicas regulares; e 11,6\% no grupo de apoio psicológico e motivacional). Isoladamente estes indicadores são bastante limitados, mas o ML permitiu ainda a identificação de aspectos dificultadores e facilitadores para a adesão e implantação do programa nessa comunidade.

A falta de profissionais de educação física e nutrição no NASF; dificuldades para encontrar locais com acesso livre que comportassem grandes grupos; a desmotivação parcial dos ACS para desenvolver atividades regulares do programa; e a falta de comunicação entre os profissionais, levando ao desenvolvimento de ações sobrepostas realizadas de forma isolada, foram identificadas como barreiras. O financiamento através de editais, a interação do serviço de saúde com o meio acadêmico, a motivação apresentada pelo GG e pelos usuários do serviço, e a interação social com a comunidade foram destacados como facilitadores da implantação e da adesão.

\section{DISCUSSÃO}

A elevada prevalência de fatores de risco para DCNT no DF ${ }^{3,25}$ reforça a importância de desenvolver ações de PS e legitima a execução do programa Jogo de Cintura. A relevância científica deste artigo reside justamente na descrição e divulgação do ML de um programa de PS piloto no DF, indicando o percurso realizado no decorrer de sua implantação. Esta experiência pode ser útil tanto como um modelo a ser seguido, como também apontando caminhos para o planejamento de intervenções na rede pública adequadas à realidade local, encorajando pesquisadores, profissionais e gestores da saúde a se dedicar a esta importante temática. Ademais, para realizar uma avaliação adequada de programas de PS, é fundamental que se registre o processo de implantação a partir da realidade encontrada ${ }^{9,10,15}$. Esta fase que precede a avaliação propriamente dita é indispensável para entender a dinâmica da iniciativa e crucial para nortear modificações e melhoras em sua implementação, favorecendo a efetividade, sustentabilidade e expansão $0^{4,9,10}$. Nesta perspectiva, cabe destacar a intenção da SES-DF de monitorar continuamente e implantar o programa Jogo de Cintura em todas as regiões administrativas do DF.

Outras lições aprendidas decorrentes da implantação do Jogo de Cintura e de sua sistematização no ML merecem ser compartilhadas. Quanto à mobilização das partes interessadas, pode-se perceber que a identificação e fixação de parcerias 
estratégicas foi fundamental para o desenvolvimento do programa. A constituição do GG com participação de gestores, profissionais e pesquisadores de diferentes áreas da saúde possibilitou análises contextuais múltiplas e aprofundadas de todas as etapas do processo. $\mathrm{O}$ entendimento e a descrição clara dos objetivos, metas e resultados esperados com o programa foi uma etapa difícil mas crucial para organizar as ações subsequentes, permitindo adequá-las ao contexto social específico da região, aumentando as chances de sustentabilidade do programa ${ }^{6,14}$.

Para escolher a área que recebeu a versão piloto do programa, foram utilizados indicadores disponíveis como: elevada prevalência de comportamentos de risco ${ }^{25}$; menor IDH indicando adversidades sociais, econômicas e estruturais na comunidade ${ }^{24}$, que apresentam relação com fatores de risco para DCNT (obesidade, escolhas alimentares inadequadas e má utilização do tempo de lazer) ${ }^{3,28}$; e a amplitude da cobertura da ESF, que está diretamente relacionada à estrutura de serviço existente. Todos estes foram aspectos fundamentais para justificar e manter o programa ${ }^{23}$. Após, a escolha do público alvo ocorreu pela "abordagem de risco", uma forma relativamente fácil de identificar sujeitos com as características de risco desejadas nos serviços de saúde, bem como com maior potencial de adesão devido ao interesse em melhorar sua saúde ${ }^{15}$.

Os procedimentos e equipamentos utilizados foram simples e baratos e, conforme recomendado, foram adequados à realidade local ${ }^{9,15}$. É essencial reconhecer ainda o papel central dos profissionais que atuam nas ESF no fortalecimento do compromisso dos usuários com o programa. Salienta-se entretanto, que a quantidade e a diversidade de profissionais atuantes foi insuficiente para otimizar as intervenções comportamentais em nível populacional. $\mathrm{O}$ aspecto interdisciplinar destas intervenções pressupõe uma equipe mais completa (com nutricionistas e profissionais de educação física, por exemplo), com tempo para reflexão conjunta sobre os aspectos bio-psico-sociais que compõem o indivíduo ${ }^{4,15}$.

Para desenvolver as atividades, buscou-se estratégias para o alcance das metas, divididas em objetivos de pesquisa e de $\mathrm{PS}^{10}$. Cabe destacar que na coleta de dados, os participantes, em sua maioria de baixa renda e escolaridade, apresentaram dificuldades para responder os questionários de hábitos alimentares e atividades físicas, o que terá impacto na precisão dos dados e na avaliação futura sobre o alcance destas metas. Espera-se que, com a maior discussão desta temática, maneiras mais simples e fidedignas para avaliar hábitos de vida possam ser desenvolvidas e validadas.

Em relação às intervenções, inicialmente foi feito um levantamento das atividades que já estavam em andamento nas ESF, e estas foram reforçadas ou ajustadas pelo programa, pois um dos objetivos da PNPS é integrar, de forma coordenada, as ações em PS ${ }^{1,4}$. Esta sistematização permitiu também identificar novas ações que deveriam ser desenvolvidas para garantir que os objetivos propostos fossem atingidos. Intervenções em grupos possibilitaram minimizar problemas comuns na rede pública de saúde: a grande demanda de usuários somada à composição defasada das equipes. A abordagem interdisciplinar permitiu que os profissionais e usuários compartilhassem vários olhares sobre a mesma temática, reduzindo as dúvidas. As dinâmicas motivacionais facilitaram o compartilhamento de experiências e a discussão de barreiras e estratégias para seu enfrentamento. As atividades lúdicas foram importantes para criar vínculo entre o grupo e desenvolver o senso de corresponsabilidade com a saúde entre usuários e profissionais. Cabe destacar que, mesmo assim, a perda de participantes foi aumentando com o passar do ano, chegando a mais de $50 \%$ no último mutirão de coleta de dados, um problema que 
deve ser estudado em futuros artigos.

Quanto à implantação, um elemento facilitador foi a própria elaboração do ML, que permitiu ao GG compartilhar expectativas e responsabilidades; explicitou a relevância da manutenção de alianças fortes e estratégicas para desenvolver a magnitude das intervenções ${ }^{1,2,10,14}$; e reforçou o caráter adaptativo necessário para desenvolver ações interinstitucionais eficientes ${ }^{9,10,13}$. A sensibilização e capacitação dos profissionais e a elaboração e distribuição de materiais educativos também facilitaram a execução das ações no nível comunitário ${ }^{10-15}$. A regularidade dos encontros para prática de atividades físicas e as oficinas promoveram o sentido de grupo e estimularam a motivação e participação, provavelmente por favorecer o apoio e o fortalecimento das redes sociais ${ }^{15,29}$, permitindo que os profissionais conhecessem a realidade e as expectativas locais, aspectos também relatados em outras intervenções de $\mathrm{PS}^{11-15,29}$. Como barreiras, a falta de infraestrutura e profissionais foram sérios empecilhos para o desenvolvimento das ações, e são problemas também relatados em outros estudos de base populacional ${ }^{13,14}$. No Jogo de Cintura, a elaboração do ML permitiu a troca de informações com os tomadores de decisão para superá-las ao expandir o programa à outras regiões do DF. Ciente da magnitude destas dificuldades o governo federal lançou o programa Academia da $S_{a u ́ d e}$, alocando recursos para infraestrutura e profissionais na PS.

O presente artigo apresenta a limitação de não exibir os resultados do programa; dados antropométricos, bioquímicos, de conhecimentos e de hábitos de vida serão publicados em futuros artigos. Destaca-se entretanto que, ao adotar a perspectiva avaliativa de programa comunitário, os dados deverão ser continuamente monitorados através de ações de vigilância em saúde ${ }^{4}$ e que, sem a sistematização do ML toda a etapa de avaliação ficaria prejudicada por vieses advindos de variáveis não identificadas ${ }^{9,10}$. No entanto, pode-se supor que alguns resultados positivos decorrerão do programa Jogo de Cintura, como os apontados em experiências brasileiras similares, que observaram redução de fatores de risco para DCNT ${ }^{12,14,30}$.

Sabe-se que as DCNT estão relacionadas a hábitos não saudáveis que comprometem a quantidade e qualidade de $v^{2} \mathrm{da}^{2,3}$, e que mudanças sustentáveis de comportamentos são desafios aos profissionais e órgãos de saúde mundiais ${ }^{1,2,14,15}$. Assim, é essencial unir esforços em diferentes esferas para planejar e implantar programas de PS que traduzam para as realidades locais o conhecimento adquirido no âmbito científico $^{10,15}$. Entretanto, ainda são poucos os programas desenvolvidos no nível comunitário que registram e publicam seu modelo teórico de modo a permitir comparações e análises que deem suporte ao monitoramento, avaliação e replicação ${ }^{9,13}$.

\section{CONCLUSÃO}

O processo de elaboração do ML do programa Jogo de Cintura foi baseado em uma metodologia simples que facilitou o alinhamento conceitual, o registro sistemático e a comunicação dos pontos acordados entre os parceiros. Permitiu identificar e definir os atores-chave e seus papéis; as prioridades e os objetivos; as ações e os recursos necessários; e as metas e variáveis avaliativas. A sistematização destas informações permitiu identificar a intersetorialidade como aspecto central do processo de PS e facilitou a identificação de barreiras que devem ser superadas para aumentar a sustentabilidade e reprodutibilidade do programa em realidades semelhantes. O desenvolvimento deste modelo teórico é etapa fundamental para a adequada avaliação do programa, permitindo que se defina seu custo-efetividade ao promover a saúde da população. 


\section{Agradecimentos / Financiamento}

Agradecemos às contribuições e participação das chefias da Gerência de Doenças e Agravos Não Transmissíveis - GDANT, do NVEDNT, da Gerência de Gestão da ESF, do Núcleo de Patologia da SES-DF, dos estudantes de graduação em Educação Física, Nutrição e Psicologia da Universidade de Brasília e Universidade Católica de Brasília, bem como, dos representantes da FEPECS e EMBRAPA, além das equipes de Saúde da Família e da comunidade de São Sebastião.

O estudo foi financiado pelo Ministério da Saúde e Fundação de Ensino e Pesquisa em Ciências da Saúde. Não houve conflito de interesses.

\section{Contribuições dos autores}

Todos os autores participaram da elaboração, discussão e revisão final do manuscrito. Caroline Romeiro apresentou a proposta do artigo, realizou a busca dos materiais que compõem a revisão bibliográfica, coletou e sistematizou os dados do artigo; Sarah Guerra Tinoco atualizou os dados epidemiológicos, e contextualizou o artigo na área de saúde pública, ressaltando o papel da Secretaria de Estado de Saúde do Distrito Federal; Júlia Aparecida Devidé Nogueira orientou os autores e adequou a linguagem à área da atividade física e saúde pública; Kênia Mara Baiocchi de Carvalhodestacou a parte da nutrição, relacionando as áreas de atenção e promoção em saúde e adequou a formatação às normas da revista. Todos os autores leram e aprovaram a versão final do manuscrito.

\section{REFERÊNCIAS}

1. Malta DC, Castro AM, Gosch CS, et al. A Política Nacional de PS e a agenda da atividade física no contexto do SUS. Epidemiol Serv Saúde 2009; 18:79-86.

2. Organização Pan-Americana da Saúde, Ministério da Saúde. A vigilância, o controle e a prevenção das doenças crônicas não-transmissíveis: Doenças crônicas não-transmissíveis no contexto do Sistema Único de Saúde Brasileiro. Brasília: OPAS/MS; 2005.

3. Ministério da Saúde. Secretaria de Vigilância em Saúde. Secretaria de Gestão Estratégica e Participativa. Vigitel Brasil 2011: vigilância de fatores de risco e proteção para doenças crônicas por inquérito telefônico. Brasília: Ministério da Saúde; 2012.

4. Ministério da Saúde. Secretaria de Vigilância em Saúde. Secretaria de Atenção à Saúde. Política Nacional de Promoção da Saúde. Brasília: Ministério da Saúde; 2007.

5. Ministério da Saúde. Secretaria de Atenção à Saúde. Departamento de Atenção Básica. Política Nacional de Atenção Básica. Brasília: Ministério da Saúde; 2006.

6. Ministério da Saúde. Portaria no 154 de 24 de janeiro de 2008. Cria os Núcleos de Apoio à Saúde da Família - NASF. Diário Oficial da União 2008. 04 de março de 2008.

7. Ministério da Saúde. Portaria no 719 de 07 de abril de 2011. Institui o Programa Academia da Saúde no âmbito do Sistema Único de Saúde. Disponível em: http://portal.saude.gov.br/ portal/arquivos/pdf/portaria_academia_saude_719.pdf. Acessado em: 19 de janeiro de 2012.

8. Agência Nacional de Vigilância Sanitária. Agenda Nacional de Prioridades de Pesquisa em Vigilância Sanitária. Brasília: Núcleo de Educação, Pesquisa e Conhecimento - NEPEC/ ANVISA, 2011.

9. U.S. Department of Health and Human Services. Manual para Avaliação de Programas de Atividade Física. Atlanta: U.S. Department of Health and Human Services, Centers for Disease Control and Prevention; 2002.

10. Souza EMD. Evaluation methods in health promotion programmes: the description of a triangulation in Brazil. Ciência \& Saúde Coletiva, 2010; 15(5):2521-32.

11. Matsudo V, Matsudo S, Andrade D, et al. Promotion of physical activity in a developing country: The Agita São Paulo experience. Public Health Nutr 2002; 5:253-261.

12. Simões EJ, Hallal P, Pratt M, et al. Effects of a Community-Based, Professionally Supervised Intervention on Physical Activity Levels Among Residents of Recife, Brazil. Am J Public Health 2009; 99:68-75. 
13. Ribeiro IC, Torres A, Parra DC, et al. Using Logic Model as Iterative Tools for Planning and Evaluating Physical Activity Promotion Programs in Curitiba, Brazil. J Phys Act Health 2010; 7 (S2): S155-S162.

14. Brasil. Ministério da Saúde. Secretaria de Vigilância em Saúde. Avaliação de Efetividade de Programas de Atividade Física no Brasil. Brasília: Ministério da Saúde, 2011.

15. de Barros CR, Cezaretto A, Salvador EP, et al. Implementação de programa estruturado de hábitos de vida saudáveis para redução de risco cardiometabólico. Arq Bras Endocrinol Metab 2013;57(1):7-18.

16. Governo do Distrito Federal. Secretaria de Estado de Saúde. Subsecretaria de Vigilância em Saúde. Diretoria de Vigilância Epidemiológica. Gerência de Doenças e Agravos não Transmissíveis. Plano Distrital de PS. Brasília: Secretaria de Estado de Saúde, 2007.

17. Brasil. Ministério da Saúde. Portaria no 3.228/GM, de 21 de dezembro de 2007 publicada no Diário Oficial da União- DOU no. 246.

18. World Health Organization. Fifty-seventh World Health Assembly. Agenda item 12.6. Global Strategy on Diet, Physical Activity and Health, 22 May 2004.

19. Ministério da Saúde. Guia alimentar para a população brasileira: promovendo a alimentação saudável. Série A-Normas e manuais técnicos. Brasília (DF): Ministério da Saúde. Secretaria de Atenção à Saúde. Política de Alimentação e Nutrição; 2005.

20. Klein S, Sheard NF, Pi-Sunyer X, et al. Weight management through lifestyle modification for the prevention and management of type 2 diabetes: rationale and strategies: a statement of the American Diabetes Association, the North American Association for the Study of Obesity, and the American Society for Clinical Nutrition. Diabetes Care. 2004;27(8):2067-73.

21. American Diabetes Association. Standards of medical care in diabetes-2013. Diabetes Care. 2013 Jan;36 Suppl 1:S11-66.

22. Expert panel on detection, evaluation, and treatment of high blood cholesterol in adults. Third Report of the National Cholesterol Education Program (Adult Treatment Panel III). JAMA 2001;285(19):2486-97.

23. Tinoco SGG, Leite EG, Baldini NLF. Reflexões sobre a política de PS no Distrito Federal e sua integração com a Estratégia Saúde da Família. Tempus - Actas de Saúde Coletiva 2009; 3:64-75.

24. Governo do Distrito Federal [homepage]. Índice de desenvolvimento Humano por Região Administrativa - Distrito Federal - 2000. Disponível em http://www.distritofederal.df.gov. br/005/00502001.asp?ttCD_CHAVE=1621. Acessado em: 07 de maio de 2011.

25. Yokota RTC, Vasconcelos TF, Ito MK, et al. Prevalência de fatores de risco para doenças crônicas não-transmissíveis em duas regiões do Distrito Federal. Com Ciências Saúde 2007; 18(4):289-296.

26. Craig CL, Marshall AL, Sjostrom M, et al. International physical activity questionnaire: 12-country reliability and validity. Med Sci Sports Exerc. 2003; 35(8):1381-95.

27. World Health Organization. Diet, nutrition and the prevention of chronic diseases. Report of a joint WHO/FAO expert consultation. Geneva: World Health Organization; 2003. WHO Technical Report Series, 916.

28. Castanheira M, Olinto MTA, Gigante DP. Associação de variáveis sócio-demográficas e comportamentais com gordura abdominal em adultos: estudo de base populacional no sul do Brasil. Cad Saúde Pública 2003; 19(S1):S55-S65.

29. Mazo GZ, Meurer ST, Benedetti TRB. Motivação de idosos para a adesão a um programa de exercícios físicos. Psicol Am Lat [periódico na internet]; 2009. Disponível em: http:// psicolatina.org/18/motivacion.html. Acessado em: 04 de janeiro de 2012.

30. Gomes MA, Duarte MFS. Efetividade de uma intervenção de atividade física em adultos atendidos pela estratégia Saúde da Família: Programa Ação e Saúde Floripa - Brasil. Rev Bras Ativ Fís Saude 2008; 13:44-56.

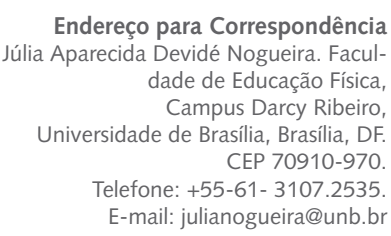

Endereço para Correspondência Júlia Aparecida Devidé Nogueira. Faculdade de Educação Física, Campus Darcy Ribeiro, Universidade de Brasília, Brasília, DF CEP 70910-970. Telefone: $+55-61-3107.2535$ E-mail: julianogueira@unb.br

Recebido 18/02/2013

Revisado 04/03/2013 Aprovado 10/04/2013 